\title{
American Board of Family Medicine In-Training Examination
}

The American Board of Family Medicine's (ABFM) In-Training Examination was conceived in 1979 as part of a tripartite assessment process for Family Medicine residents in training. This 3-fold assessment process was developed under the aegis of the Conjoint Committee on In-Training Assessment (CONCITA), a group consisting of members from the American Academy of Family Physicians, the Society of Teachers of Family Medicine, and the then American Board of Family Practice. At that time, CONCITA had envisioned moving forward with the formulation of criteria for assessing psychomotor (procedural) skills and a methodology for assessing interpersonal skills and attitudes (behavioral). The cognitive examination, first given with great success in 1979, and again each year thereafter, remains as the only vestige of this early work on resident assessment within our specialty.

Last year, the ABFM conducted a Pilot Project for its delivery of the In-Training Examination (ITE) directly to volunteer programs over the internet. The purposes of the Pilot Project included the development of administrative relationships with program coordinators required for the successful implementation of the examination delivery over the Internet, as well as feedback from those program coordinators and residents. In addition, the Pilot Project allowed for determination of the range of technical requirements necessary for working with multiple residency programs and the impact of delivering the ITE in this manner on the ABFM's information technology infrastructure, including its broadband capacity and Web servers.

A total of 633 residents across 41 ACGME accredited residency programs participated in the Internet-Based ITE (IBITE) Pilot Project. In addition to the US Family Medicine residency programs, 2 international groups participated in the IBITE Pilot Project. The Hope Family Medicine Residency Program, located in Macau, had 4 physicians take the examination. The Australian
College of Rural and Remote Medicine had 12 physicians from various geographic regions take the examination. The administration of the IBITE went very smoothly, with only minor difficulties arising which were cleared up in minutes with the assistance of the ABFM support staff. The summary statistical data comparing results of the written ITE with the Internet-Based ITE showed no significant differences in performance. The findings support the position that the ITE can be successfully and effectively administered via the Internet.

The ABFM has consistently underwritten some of the costs of the development and administration of the ITE over the years. This was in recognition of the economic factors affecting residency programs and their residents and the desire of the ABFM to limit any economic obstacles to participation in this valuable process. The fee has remained constant at \$30 per resident since 1995 . Our direct and indirect development costs of the ITE examination and the resulting scoring and reporting costs have consistently exceeded the amount collected.

The inflationary increase in the ABFM's costs, plus the development and maintenance costs of the ITE, have necessitated that we raise the fee for the ITE beginning in 2007. The 2007 fee will be $\$ 50$ per resident. Although this is a substantial increase over the current fee, it still does not allow for the ABFM to fully recover its costs. Nevertheless, the ABFM believes that the fee fairly reflects the improved efficiencies achieved in registering residents using the Resident Training Management (RTM) software; the advent of electronic reporting of ITE results to programs, to be consistent with how Certification Performance Reports are presently made available; and, the new Resident's Portfolio, which next year will allow residents to access their results directly.

All residents who have been entered in RTM have free availability of all the components of the 
ABFM's program for Maintenance of Certification for Family Physicians (MC-FP). While RTM has streamlined the process of registering residents for the In-Training and Primary Certification Exams, it has also created the ability to make MC-FP modules developed by the ABFM accessible online for residents. The ABFM believes that the residency program directors and residents will find these to be valuable resources to assist with the achievement of many of the 6 ACGME general competencies, which are the same competencies used to assess family physicians in MC-FP. 